\title{
MAPK inhibitors protect against early-stage osteoarthritis by activating autophagy
}

\author{
CHUN-NA LAN ${ }^{1}$, WEI-JUN CAI ${ }^{2}$, JIE SHI $^{3}$ and ZHONG-JIE YI ${ }^{4}$ \\ ${ }^{1}$ Department of Rehabilitation, The Second Xiangya Hospital of Central South University, Changsha, Hunan 410011; \\ ${ }^{2}$ Department of Histology and Embryology, School of Basic Medicine, Central South University; \\ ${ }^{3}$ Department of General Surgery, The Third Xiangya Hospital of Central South University, Changsha, \\ Hunan 410013; ${ }^{4}$ Department of Burn and Plastic Surgery, The Second Xiangya Hospital of \\ Central South University, Changsha, Hunan 410011, P.R. China
}

Received December 4, 2020; Accepted September 1, 2021

DOI: $10.3892 / \mathrm{mmr} .2021 .12469$

\begin{abstract}
Osteoarthritis (OA) is a chronic, age-related osteoarthropathy that causes a considerable decline in quality of life, as well as economic losses due to its high incidence and poor prognosis. Mitogen-activated protein kinases (MAPKs) regulate multiple cellular processes, including proliferation, differentiation and apoptosis, in certain diseases, such as cancer, diabetes and Alzheimer's disease. The present study aimed to investigate the regulatory role of the MAPK signaling pathway in early-stage OA. A rabbit model of early-stage OA was induced by treatment with the enzyme papain. U0126 [an extracellular signal-regulated kinase (ERK) inhibitor], SP600125 [a Jun NH2-terminal kinase (JNK) inhibitor] and SB203580 (a p38 inhibitor) were administered to the rabbits via intra-articular injection. The severity of OA was assessed by histological examination using $\mathrm{H} \& \mathrm{E}$, toluidine blue and safranin-O/fast green staining, as well by analyzing the glycosaminoglycan (GAG) content and determining the OA Research Society International (OARSI) score. Western blotting was used to detect the protein expression levels of
\end{abstract}

Correspondence to: Dr Zhong-Jie Yi, Department of Burn and Plastic Surgery, The Second Xiangya Hospital of Central South University, 139 Renmin Road, Changsha, Hunan 410011, P.R. China E-mail: yizhongjie312@csu.edu.cn

Dr Jie Shi, Department of General Surgery, The Third Xiangya Hospital of Central South University, 138 Tongzipo Road, Changsha, Hunan 410013, P.R. China

E-mail: shijie3451@csu.edu.cn

Abbreviations: MAPK, mitogen-activated protein kinase; OA, osteoarthritis; MMP3, matrix metalloproteinase-3; GAG, glycosaminoglycan; ULK1, UNC-51-like kinase 1; LC3, microtubule-associated protein 1 light chain 3; ERK, extracellular signal-regulated kinase; JNK, Jun NH2-terminal kinase; OARSI, Osteoarthritis Research Society International

Key words: osteoarthritis, MAPKs, autophagy, JNK, ERK, MMP3 matrix metalloproteinase-3 (MMP3), ERK, phosphorylated (p)-ERK, p38, p-p38, JNK, p-JNK, Beclin1, UNC-51-like kinase 1 (ULK1) and microtubule-associated protein 1 light chain 3 (LC3)II/I. U0126, SP600125 or SB203580 treatment significantly decreased the OARSI scores and significantly increased the GAG levels in the cartilaginous tissues of OA model rabbits. These results indicated that the MAPK inhibitors reduced the severity of OA-induced injury at the early stage. Western blotting results demonstrated that MAPK inhibition significantly decreased the protein expression levels of MMP3 in OA cartilage. The protective effect of MAPK inhibitors in OA was mediated via the activation of autophagy, as demonstrated by the increased protein expression levels of LC3II/I, ULK1 and Beclin1. Overall, the data indicated that MAPK inhibitors may exert a protective effect against OA by restoring compromised autophagy. Furthermore, the present study suggested that MAPK inhibitors may represent a potential pharmacological strategy for treating OA in the future.

\section{Introduction}

Osteoarthritis (OA) is a chronic, age-related osteoarthropathy characterized by loss of cartilage, remodeling of subchondral bone, formation of articular marginal osteophytes and the presence of synovitis $(1,2)$. OA in joints is a major cause of deformity, pain and disability, which results in considerable socioeconomic costs worldwide due to its high incidence $(3,4)$. Degeneration of the cartilage structure is associated with changes in the extracellular matrix (ECM), including matrix metalloproteinase-3 (MMP3), collagen and glycosaminoglycan (GAG), which are all regarded as biological markers of OA (5). The severity of OA is correlated with MMP3 accumulation and GAG reduction. OA can be induced by various physical and biochemical triggers $(6,7)$, with pathological changes being irreversible at the middle and late stages of the disease. Therefore, it is important to develop therapeutic strategies to treat the early stage of the disease (8).

Autophagy is a metabolic process in which cytoplasmic organelles and cytosolic components are degraded to protect cells from stress, and it is a mechanism that is present in numerous diseases (9-12). The expression levels of UNC-51-like 
kinase 1 (ULK1), Beclin1 and microtubule-associated protein 1 light chain 3 (LC3), which function as the initiator, regulator and executor of autophagy, respectively, are commonly used as a biomarker of the autophagic process (13). Autophagy has been considered to serve as a protective mechanism in maintaining normal cartilage function, and compromised autophagy has been demonstrated to lead to OA and numerous other age-related diseases (14-18). Therefore, targeting autophagy may serve as a promising therapy for OA.

Mitogen-activated protein kinases (MAPKs) are a family of Ser/Thr protein kinases thar are widely found in eukaryotes and include extracellular signal-regulated kinase (ERK), p38 and c-Jun NH2-terminal kinase (JNK). MAPKs regulate multiple cellular processes, such as proliferation, differentiation, survival and apoptosis $(19,20)$. Previous studies have demonstrated that JNK and p38 contribute to apoptosis, whereas ERK mediates proliferation in diseases such as OA, cancer, diabetes and Alzheimer's disease (19,21-23). However, the role of MAPKs in autophagy varies among diseases (24-31). Previous studies have also reported that p38 acts synergistically with autophagy, whereas other studies have demonstrated that MAPKs attenuate autophagy under certain conditions (32-34). The effect of MAPKs on autophagy in the pathogenesis of OA remains unclear.

In our previous study, compromised autophagy was demonstrated to be closely related to the progression of OA, and JNK and p38 MAPKs increased MMP3 expression and inhibited autophagy (18). Selective inhibition of the MAPK/MMP3 signaling pathway and restoration of impaired autophagy may serve as a novel therapeutic strategy for OA and other degenerative disorders.

In the present study, the effects of MAPK inhibitors on autophagy in a rabbit model of early-stage OA, using intra-articular injections of U0126 (an ERK inhibitor), SP600125 (a JNK inhibitor) and SB203580 (a p38 inhibitor), were explored.

\section{Materials and methods}

Ethics. All animal experiments were approved by the Institutional Animal Care and Use Committee of The Second Xiangya Hospital of Central South University (Changsha, China; approval no. 2018005). All experimental procedures were performed in accordance with the Animal Research: Reporting of in vivo Experiments guidelines (35) and the National Institutes of Health Guide for the Care and Use of Laboratory Animals (36).

Animals. Healthy male New Zealand rabbits (age, 4 months; weight, $2 \mathrm{~kg}$; purchased from Hunan Pacific Biological Technology Co., Ltd.) were used in the present study. The animals $(n=40)$ were randomly assigned into the following five groups: i) the control group; ii) the OA group; iii) the $\mathrm{OA}+\mathrm{U} 0126$ group; iv) the OA + SP600125 group; and v) the $\mathrm{OA}+\mathrm{SB} 203580$ group. In the control group, the animals received an articular injection of $0.9 \% \mathrm{NaCl}(0.1 \mathrm{ml})$ once a day for 3 consecutive days. In the OA groups, an articular injection of $0.1 \mathrm{ml} 4 \%$ papain enzyme was administered once a day for 3 consecutive days to establish the respective OA model. For the OA + U0126, OA + SP600125 and OA + SB203580 groups, at 7 days after the last injection of papain, animals received an intra-articular injection of $0.1 \mathrm{ml}(100 \mu \mathrm{mol} / \mathrm{l})$ of U0126 (an ERK inhibitor; Abcam; cat. no. ab120241), SP600125 (a JNK inhibitor; Abcam; cat. no. ab120065) or SB203580 (a p38 inhibitor; Abcam; cat. no. ab120162), respectively, once a day for 3 days. All animals were fed in cages at the Animal Center of Second Xiangya Hospital with a 12-h light/dark cycle, with free access to water and standard chow, and were monitored every day. The environmental temperature was maintained at $20-25^{\circ} \mathrm{C}$ and the relative humidity was $50 \pm 5 \%$. Animals were euthanized at 15 days after the last intra-articular injection of papain by $20 \mathrm{ml} / \mathrm{kg}$ of air embolism under anesthesia $(30 \mathrm{mg} / \mathrm{kg}$ of $3 \%$ pentobarbital sodium via the ear vein). Cartilage of the medial condyles of the femur was then removed for general observation, histological examination and western blotting. Changes in rabbit weight were calculated before and after the experiment.

General observation of $O A$ severity. The distal epiphyses of the femurs were collected following animal euthanasia and washed in normal saline at room temperature. For the macroscopic observations, photographs of the rabbit knees were recorded using a digital camera from the same distance for each sample, and descriptive methods were used for examining the thickening of cartilage, cartilage rough surface and exudation of synovial fluid that were observed by the naked eye. OA cartilage was characterized by joint swelling, cartilage hyperemia, thickening of cartilage, a rough cartilage surface and the abundant exudation of synovial fluid.

Histology. The medial condyles of the femurs were fixed in $4 \%$ paraformaldehyde solution for $24 \mathrm{~h}$ and then decalcified in $11 \%$ EDTA disodium buffer. The buffer was replaced every 3 days until the cartilage was completely decalcified after 15 days. The condyles were then dehydrated and embedded in paraffin. Frontal serial sections (thickness, $6 \mu \mathrm{m}$ ) were cut and mounted on polysine adhesion glass slides. Toluidine blue staining, H\&E staining and safranin-o/fast green staining were performed to evaluate morphological changes in chondrocytes. Sections were observed using a light microscope. All the procedures carried out at room temperature.

To evaluate the pathological changes of early-stage OA, cartilage was subjected to histological examination of lesion depth, lesion extent over cartilage surface, matrix staining, chondrocyte clustering, surface fibrillation and abrasion quantification of GAG. For semi-quantitative analysis of GAG, Image-Pro Plus version 6 (Media Cybernetics, Inc.) software was used to measure the average optical density of the toluidine blue staining (at wavelength of $255 \mathrm{~nm}$ ). Five randomly selected fields of view were analyzed from each slice and the average optical density of each group was compared. The severity of OA was evaluated based on the OA Research Society International (OARSI) scoring system as previously described $(35,36)$.

Western blotting. Total protein was extracted from the medial condyles in lysis buffer (Cell Signaling Technology, Inc.) and used to detect the protein expression levels of phosphorylated (p)-ERK, ERK, p-p38, p38, p-JNK, JNK, MMP3, Beclin1, ULK1 and LC3B (including LC3-II and LC3-I). Total protein 
concentration was quantified using the BCA Kit (Thermo Fisher Scientific, Inc.). Equal amounts of protein $(20 \mu \mathrm{g})$ were separated via $10 \%$ SDS-PAGE and transferred onto PVDF membranes via electroblotting. The membranes were blocked for $1 \mathrm{~h}$ at room temperature in 5\% BSA solution (cat. no. 9048-46-8; GENVIEW) in $1 \mathrm{~mol} / 1$ TBS-Tween 20 [TBST; pH 7.5; 0.1\% (v/v) Tween-20]. The membranes were subsequently incubated overnight at $4{ }^{\circ} \mathrm{C}$ with the following primary antibodies: Anti- $\beta$-actin (1:5,000; cat. no. ab8227; Abcam), anti-ERK (1:500; cat. no. bs-0022R; BIOSS), anti-p38 (1:500; cat. no. bs-0637R; BIOSS), anti-JNK (1:500; cat. no. bs-2900R; BIOSS), anti-p-ERK (1:500; cat. no. bs-3016R; BIOSS), anti-p-p38 (1:500; bs-0636R; BIOSS), anti-p-JNK (1:1,000; cat. no. ab124956; Abcam), anti-ULK1 (1:1,000; cat. no. bs-3602R; BIOSS), anti-LC3B (1:500; cat. no. NB100-2220; Novus Biologicals, LLC), anti-Beclin1 (1:1,000; cat. no. LS-C117076; LifeSpan Biosciences, Inc.) and anti-MMP3 (1:500; cat. no. 17873-1-AP; ProteinTech Group, Inc.). To visualize the bands following the primary incubation, the membranes were incubated with horseradish peroxidase-conjugated secondary antibodies $(1: 6,000$; cat. no. SA00001-2; ProteinTech Group, Inc.) for $1 \mathrm{~h}$ at room temperature. Membranes were washed three times, for $10 \mathrm{~min}$ each time, in $1 \mathrm{~mol} / \mathrm{l}$ TBST after incubation with the primary and secondary antibodies. Protein bands were visualized using chemiluminescence (Thermo Fisher Scientific, Inc.). Grayscale densitometry analysis was performed using Image Pro-Plus version 6 software with $\beta$-actin as the loading control.

Statistical analysis. GraphPad Prism 7.0 (GraphPad Software, Inc.) was used to analyze the data. Analysis of three or more independent groups was performed by one-way ANOVA followed by Tukey's post hoc test. $\mathrm{P}<0.05$ was considered to indicate a statistically significant difference. Experiments were repeated $\geq 3$ times and all animals were included in the analysis to avoid biased parameter estimates.

\section{Results}

General observation of OA severity. Table I displays the average body weight of the animals in each group before the study and at the time of sacrifice. Within each group, the change in body weight between the two time points was $<0.5 \mathrm{~kg}$, which was not statistically significant. Compared with the healthy cartilage in the control group, the cartilage in the OA group exhibited joint swelling, cartilage hyperemia, thickening of cartilage, rough cartilage surfaces and abundant exudation of synovial fluid. The severity of OA was markedly reduced in animals receiving an intra-articular injection of U0126, SP600125 or SB203580 compared with that in the OA group (Fig. 1).

U0126, SP600125 and SB203530 ameliorate OA severity. Histological OA damage was evaluated by $\mathrm{H} \& \mathrm{E}$, toluidine blue and safranin-o/fast green staining. Cartilage from the OA group exhibited pathological changes characterized by thickening of the cartilage surface, cell disorientation, cell clustering and uneven matrix distribution compared with the control group. However, these pathological changes were markedly attenuated in OA model animals treated with the MAPK inhibitors U0126, SP600125 and SB203530 (Fig. 2A-C).
Table I. Changes in rabbit weight before and after the experiment.

\begin{tabular}{lcc}
\hline Group $(\mathrm{n}=8)$ & $\begin{array}{c}\text { Before study, } \\
\mathrm{kg}\end{array}$ & $\begin{array}{c}\text { After sacrifice, } \\
\mathrm{kg}\end{array}$ \\
\hline Control & $1.93 \pm 0.05$ & $2.36 \pm 0.08$ \\
OA & $1.99 \pm 0.05$ & $2.38 \pm 0.07$ \\
OA + U0126 & $2.00 \pm 0.06$ & $2.44 \pm 0.07$ \\
OA + SP600125 & $2.01 \pm 0.06$ & $2.39 \pm 0.08$ \\
OA + SB203580 & $2.00 \pm 0.06$ & $2.39 \pm 0.03$ \\
\hline
\end{tabular}

Data are presented as the mean $\pm \mathrm{SD}$. Each of the two groups were compared using a paired Student's t-test. Results were not statistically significant $(\mathrm{P}>0.05)$. OA, osteoarthritis.

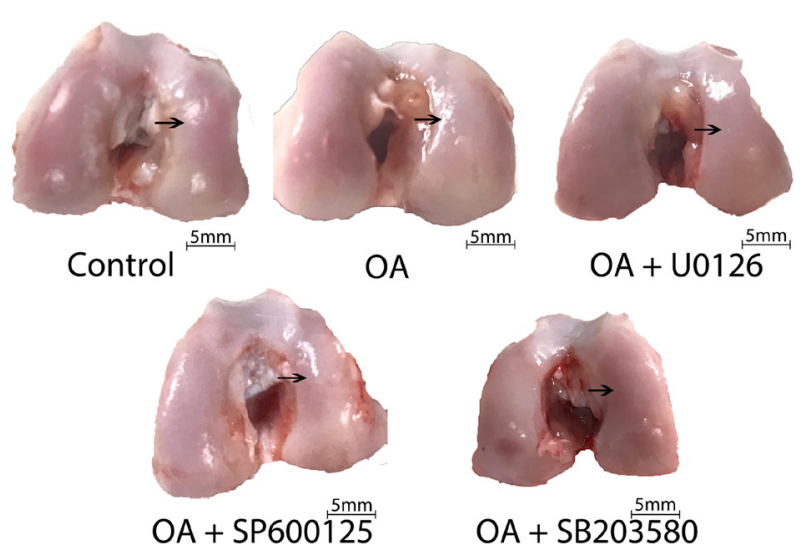

Figure 1. General observation of OA severity. Compared with the healthy cartilage in the control group, the cartilage in the OA group exhibited joint swelling, cartilage hyperemia, thickening of cartilage, rough cartilage surfaces and abundant exudation of synovial fluid. The severity of OA was markedly reduced in animals receiving an intra-articular injection of U0126, SP600125, or SB203580 compared with that in the OA group. Scale bar, $5 \mathrm{~mm}$. OA, osteoarthritis. Arrows were showed the thickening of cartilage and rough cartilage surfaces.

Semi-quantitative analysis of GAG was performed based on the optical density of the toluidine blue stain and was analyzed using Image-Pro Plus. The GAG staining in the OA group was significantly lower compared with that in the control group. However, the OA-induced decreases in GAG staining were significantly inhibited by U0126, SP600125 and SB203530 treatment (Fig. 2D). The OARSI scoring system was used to assess the severity and extent of OA based on H\&E staining and safranin-O/fast green staining. The results demonstrated that U0126, SP600125 and SB203530 treatment significantly ameliorated the severity of OA compared with that in the OA group (Fig. 2E).

Inhibition of the ERK signaling pathway by U0126 restores autophagy in $O A$. Western blotting was used to analyze protein expression levels in cartilaginous tissues (Fig. 3). p-ERK protein expression levels were significantly increased in OA animals compared with those in the control group. Moreover, the OA-induced increase in p-ERK protein expression levels was significantly reduced by the 
A

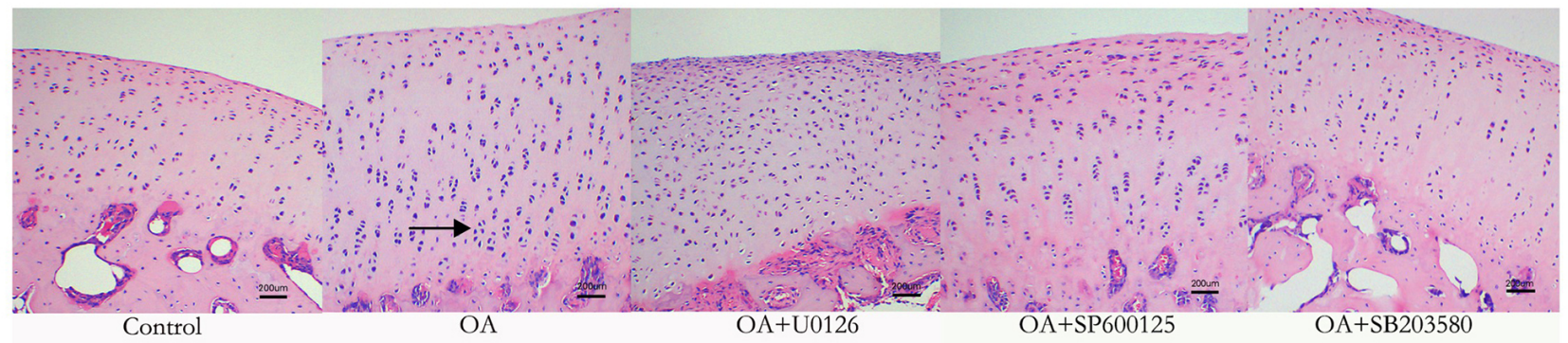

B

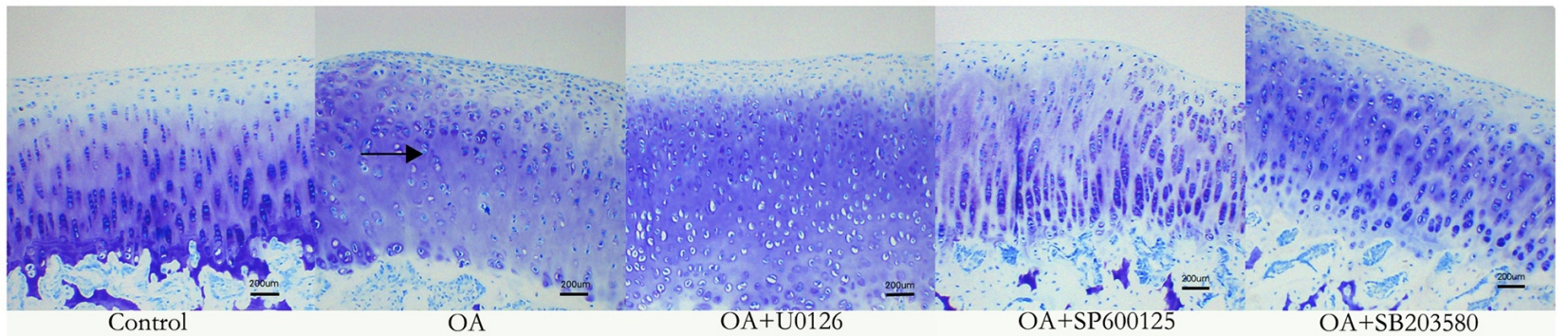

C

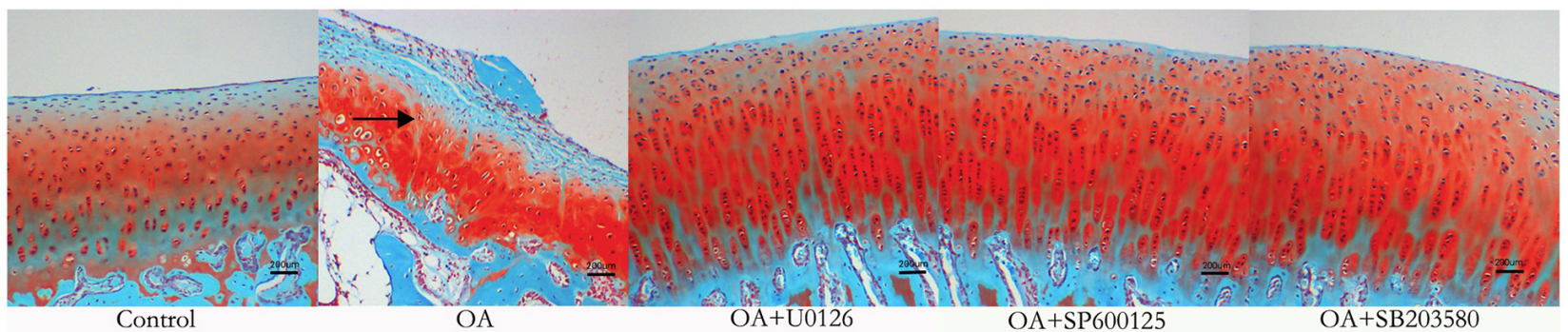

$\mathrm{D}$

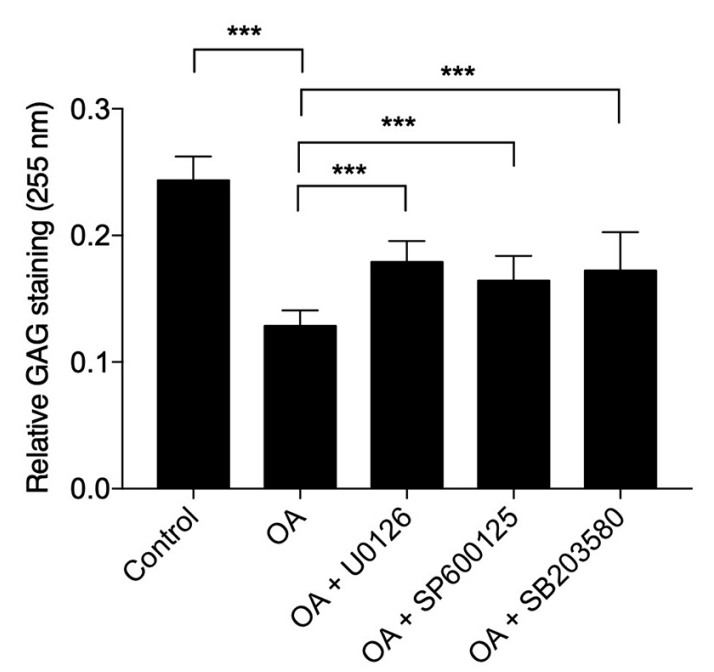

$\mathrm{E}$

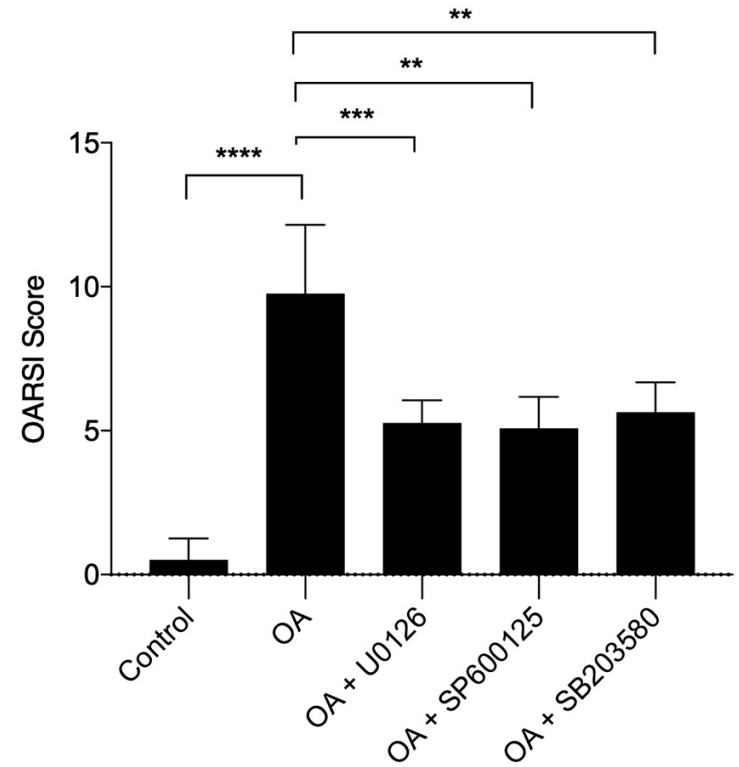

Figure 2. Histological observation of OA severity. (A) H\&E, (B) toluidine blue and (C) safranin-o/fast green staining were used to determine OA severity (magnification, x10). OA changes included chondrocyte disorientation, loss of extracellular matrix and fibrillation and fissure formation. (D) Semi-quantitative analysis of GAG staining using the mean optical density of toluidine blue staining. (E) OA severity was evaluated using the OARSI scoring system. Arrows in (A) and (B) showed the cluster and disorientation of chondrocytes. Arrow in (C) showed the loss of extracellular matrix and fibrillation and fissure formation. Data are presented as the mean $\pm \mathrm{SD} .{ }^{* *} \mathrm{P}<0.01,{ }^{* * * *} \mathrm{P}<0.001$ and ${ }^{* * * * *} \mathrm{P}<0.0001$. OA, osteoarthritis; GAG, glycosaminoglycan; OARSI, Osteoarthritis Research Society International.

ERK inhibitor U0126. However, no statistically significant differences were observed in the total ERK protein expression levels among the control, OA and OA + U0126 groups. MMP3 protein expression levels were significantly increased in the OA group compared with those in the control group.
Administration of U0126 significantly suppressed MMP3 protein expression levels compared with those in the OA group, confirming that U0126 had a protective effect against OA pathogenesis. The cartilaginous tissue of OA model rabbits had significantly reduced LC3II/I protein expression 


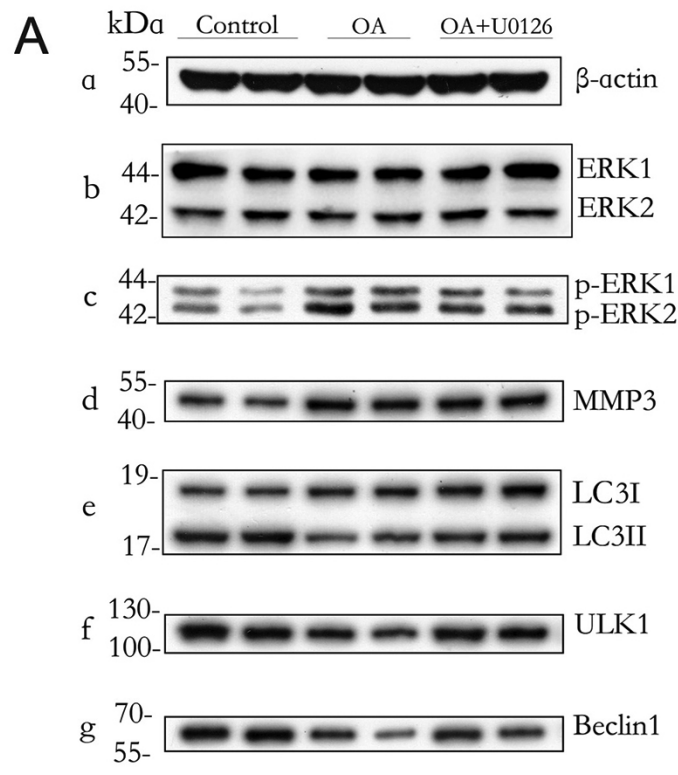

F

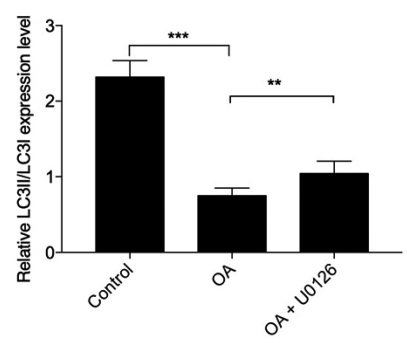

B

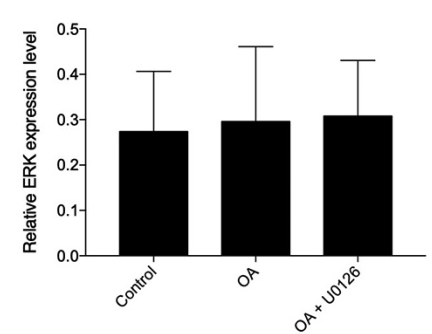

D

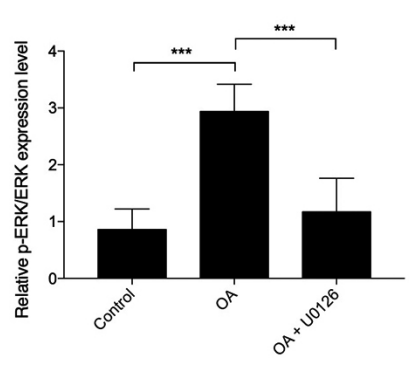

G

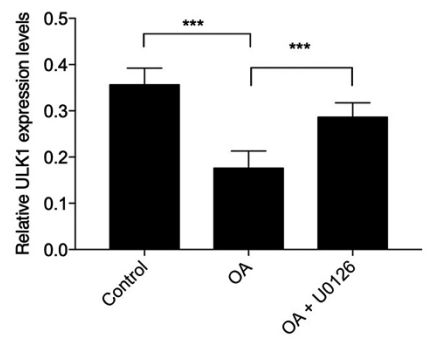

C

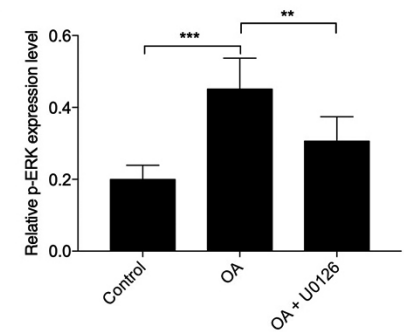

E

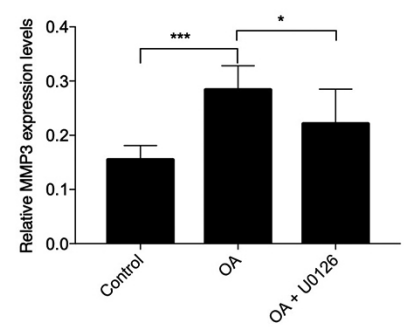

$\mathrm{H}$

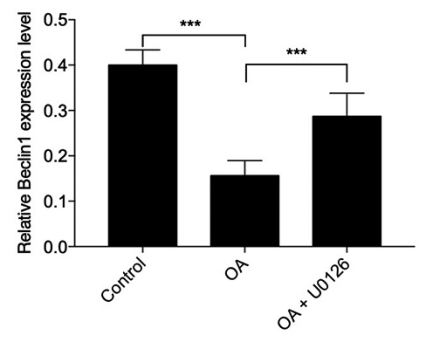

Figure 3. Effect of U0126 on autophagy in OA. Western blotting was performed to determine the protein expression levels (A-a) $\beta$-actin (loading control), (A-b) ERK, (A-c) p-ERK, (A-d) MMP3, (A-e) LC3II/LC3I, (A-f) ULK1 and (A-g) Beclin1 in cartilage lysates in each experimental group of rabbits. (B-H) Semi-quantification of band density. p-ERK protein expression levels were normalized to $\beta$-actin. $\mathrm{n}=3$. ${ }^{*} \mathrm{P}<0.05$, ${ }^{* *} \mathrm{P}<0.01{ }^{* * * *} \mathrm{P}<0.001 . \mathrm{OA}$, osteoarthritis; p, phosphorylated; ULK1, UNC-51-like kinase 1; MMP3, matrix metalloproteinase-3; LC3, microtubule-associated protein 1 light chain 3; ERK, extracellular signal-regulated kinase.

levels compared with those in the control group, which suggested that autophagy was compromised in OA. However, U0126 rescued the impaired autophagy process in OA, as demonstrated by the significantly increased LC3II/I protein expression levels in the OA + U0126 group compared with those in the OA group. U0126 treatment also significantly increased the expression of ULK1 and Beclin1 in the OA group compared with that in the control group. Overall, these results suggested that selectively blocking the ERK/ MMP3 signaling pathway may protect against compromised autophagy induced by OA.

Inhibition of the JNK signaling pathway by SP600125 restores autophagy in $O A$. Western blotting demonstrated that the protein expression levels of MMP3 and p-JNK were significantly upregulated in the OA group compared with those in the control group. These upregulated protein expression levels were significantly reduced by SP600125 treatment (Fig. 4). The significant downregulation of the protein expression levels of autophagy markers LC3II/I, ULK1 and Beclin1 in the OA group, compared with those in the control group, were significantly attenuated by SP600125 treatment. These results suggested that the protective effect of the JNK inhibitor
SP600125 against OA may be mediated via restoration of impaired autophagy in OA.

Inhibition of the p38 signaling pathway by SB203580 restores autophagy in $O A$. Western blotting demonstrated that the protein expression levels of MMP3 and p-JNK were significantly increased in the OA group compared with those in the control group. This effect was significantly decreased by SB203580 treatment (Fig. 5). The protein expression levels of LC3II/I, ULK1 and Beclin1 were significantly downregulated in the OA group compared with those in the control group, which was significantly reduced by SB203580 treatment. These results demonstrated that the selective p38 inhibitor SB203580 potentially protected against OA by positively regulating autophagy in cartilaginous tissues with $\mathrm{OA}$.

\section{Discussion}

OA is highly prevalent and has become a leading cause of disability (37). The healthcare resources and costs associated with managing OA can be substantial. Early intervention with the purpose of preventing disease onset and minimizing its progression is of vital importance $(4,6)$. In the present 

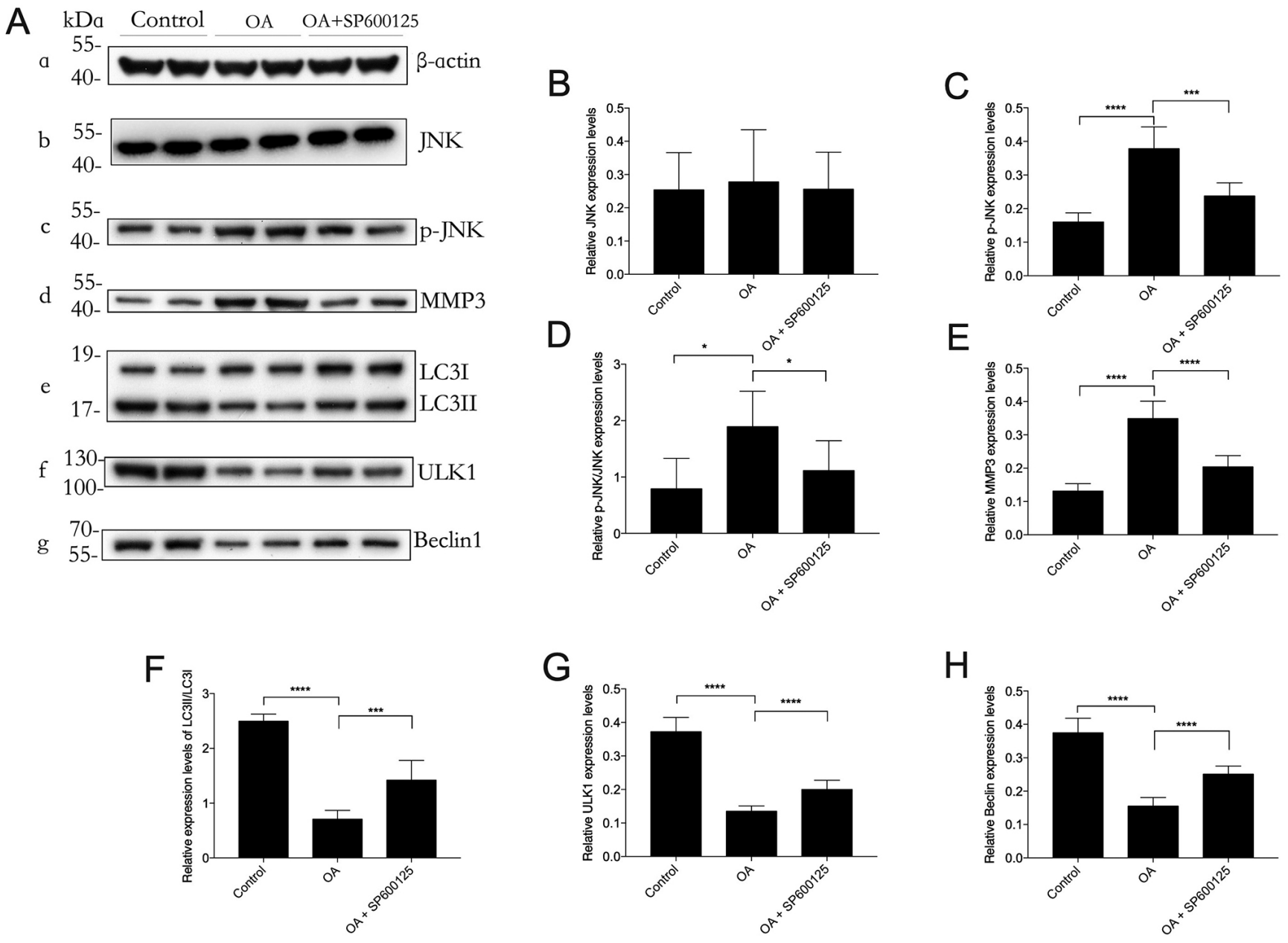

Figure 4. Effect of SP600125 on autophagy in OA. Western blotting was performed to determine the protein expression levels (A-a) $\beta$-actin (loading control), (A-b) JNK, (A-c) p-JNK, (A-d) MMP3, (A-e) LC3II/LC3I, (A-f) ULK1 and (A-g) Beclin1 in cartilage lysates in each experimental group of rabbits. (B-H) Semi-quantification of band density. p-JNK protein expression levels were normalized to $\beta$-actin. $n=3 .{ }^{*} \mathrm{P}<0.05^{* * * *} \mathrm{P}<0.001$ and ${ }^{* * * *} \mathrm{P}<0.0001$. OA, osteoarthritis; p, phosphorylated; ULK1, UNC-51-like kinase 1; JNK, Jun NH2-terminal kinase; MMP3, matrix metalloproteinase-3; LC3, microtubule-associated protein 1 light chain 3 .

study, a rabbit model of early-stage OA was established via intra-articular injection of papain, and papain is a canonical reagent for inducing OA model $(38,39)$. The current results indicated that early-stage OA could be characterized by joint swelling, cartilage hyperemia, cartilage thickening, rough cartilage surfaces and abundant exudation of synovial fluid. Although cartilage lesions could be easily identified in the early stages, we found there were no obvious symptoms or behavioral changes observed in the rabbits. The successful establishment of the OA model was further confirmed by subsequent histopathological assessment and the presence of chondrocyte proliferation, cell disorientation, loss of ECM, cartilage fibrillation and fissure formation. The OARSI scoring system was first proposed by Pritzker et al (40) in 2006 and is a useful methodology for assessing histopathological changes in OA cartilage. In contrast to the Mankin grading system (41), which primarily reflects the late stages of OA, the OARSI system, which is based on six grades reflecting the depth of the lesion and four stages reflecting the extent of OA over the joint surface, can identify differences in early or mild OA. An OARSI score $<12$ is considered to indicate early-stage OA. In 2016, a new OARSI grading system was established that reflects the differences in cartilage stiffness between OARSI grade 0 (intact surface and no signs of degeneration) and grade 1 (intact surface and early signs of arthritis) (42). Based on this new scoring system and our previous findings (18), the OARSI score in the rabbit OA model on the 15 th day after papain injection was determined to be $\sim 12$. Therefore, in the present study, rabbits were euthanized and cartilage was obtained at this time point for all experiments. The results demonstrated that early-stage OA was successfully established in rabbits. Moreover, the administration of MAPK pathway inhibitors (U0126, SP600125, or SB203580) significantly ameliorated OA-induced injury, as demonstrated by significantly diminished OARSI scores and significantly increased GAG levels.

Autophagy is an evolutionarily conserved catabolic process required for cellular homeostasis that determines cell viability in response to stress. Autophagic systems in mammals are broadly categorized into three types: Macroautophagy, microautophagy and chaperone-mediated autophagy (43). Macroautophagy is the most common and best characterized form of autophagy in mammalian cells (9-12). The process of autophagy initiates with the 


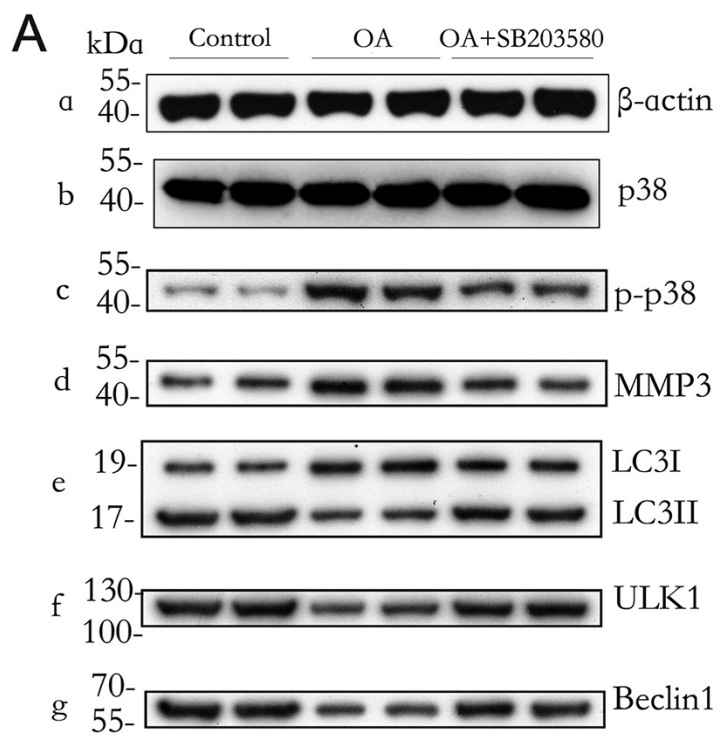

F

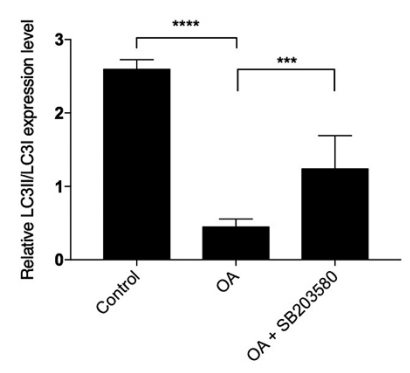

B

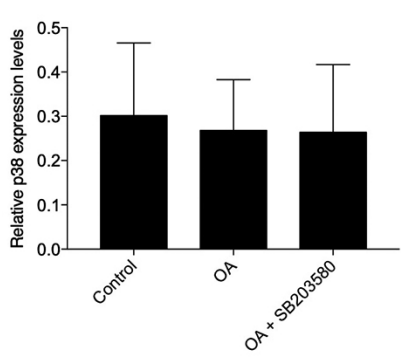

D

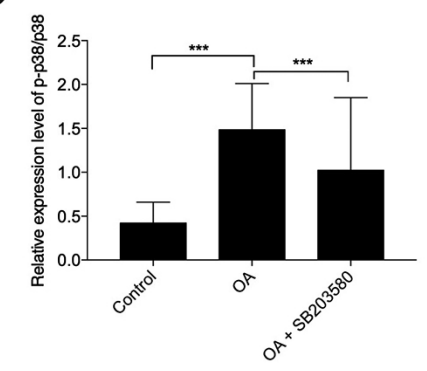

G

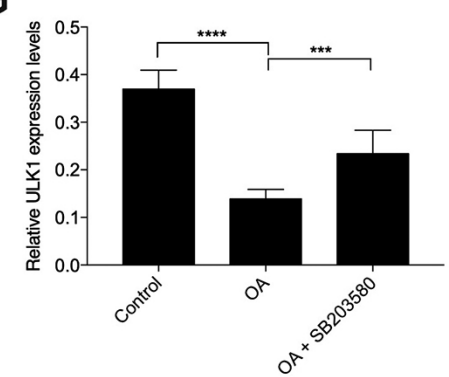

C

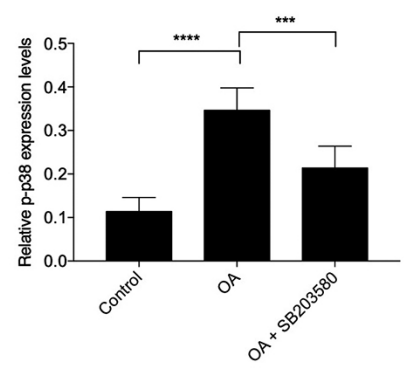

E

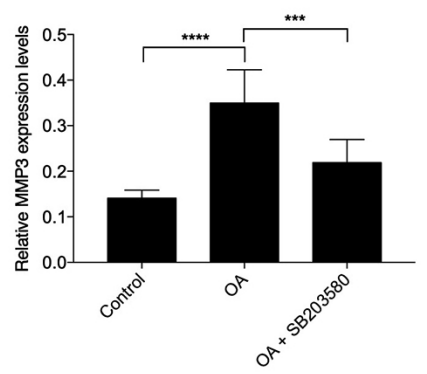

$\mathrm{H}$

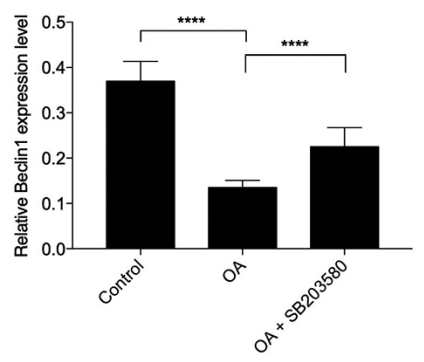

Figure 5. Effect of SB203580 on autophagy in OA. Western blotting was performed to determine the protein expression levels (A-a) $\beta$-actin (loading control), (A-b) p38, (A-c) p-p38, (A-d) MMP3, (A-e) LC3II/LC3I, (A-f) ULK1 and (A-g) Beclin1 in cartilage lysates in each experimental group of rabbits. (B-H) Semi-quantification of band density. p-p38 protein expression levels were normalized to $\beta$-actin. $\mathrm{n}=3$. ${ }^{* * * *} \mathrm{P}<0.001$ and ${ }^{* * * *} \mathrm{P}<0.0001$. OA, osteoarthritis; p, phosphorylated; ULK1, UNC-51-like kinase 1; MMP3, matrix metalloproteinase-3; LC3, microtubule-associated protein 1 light chain 3.

formation of the phagophore. Under stressful conditions, mTOR is inactivated, which consequently facilitates the formation of the ULK complex, namely ULK1-FAK family-interacting protein of $200 \mathrm{kDa}$-autophagy-related protein (Atg) 13. The ULK complex subsequently recruits other autophagy-related proteins, and Beclin1 actively forms a complex with vacuolar protein sorting (VPS) 34 and the associated protein VPS15 to induce the formation of a phagophore. The elongation of the phagophore is facilitated by two ubiquitin conjugation systems: i) The Atg12-Atg5-Atg16 complex; and ii) the phosphatidylethanolamine (PE)-conjugated LC3II system. LC3I is formed by the cleavage of pro-LC3 at the C-terminus by Atg 4 and is subsequently conjugated to PE with the help of Atg7 and Atg3 to form LC3II (9-12). LC3II binds to the autophagosome membrane and enables its fusion with the lysosome to form autophagolysosomes that subsequently degrade the accumulated cytosolic contents. In this way, autophagy serves an important role in cellular homeostasis and has been considered a protective mechanism in maintaining the function of cartilage (14-18). The present study demonstrated that the inhibition of ERK, JNK and p38 resulted in a significant increase in the protein expression levels of ULK1, Beclin1 and LC3II/I in OA model rabbits, which suggested that the inhibition of MAPKs exerted a protective effect against OA by restoring impaired autophagy in OA.

Numerous signaling pathways and kinases regulate autophagy, including Atg9, BCL-2, the forkhead box $\mathrm{O}$ family of transcription factors, AMP-activated protein kinase, Akt, protein kinase $\mathrm{C}$ and the MAPKs (ERK, p38 and JNK) (9,30). The MAPK cascades are intracellular transduction pathways that respond to various extracellular stimuli and control multiple fundamental cellular processes, including growth, proliferation, differentiation, motility, stress responses and cell death (20). MAPKs not only serve an important role in regulating cell death by apoptosis but have also been implicated in autophagy (30). Hence, the MAPK signaling pathway has become an area of high interest in the field of autophagy research in recent years. However, the effect of MAPKs on autophagy varies among different diseases (24-31). Our previous study of early-stage OA demonstrated that with the development of OA, the key markers of the JNK and p38 MAPK pathways were elevated and the biomarkers of autophagy were decreased, suggesting 
that JNK and p38 may negatively regulate the autophagic process but positively regulate MMP3 in OA (18). These findings are shown in the current study as well. ERK was also included in the present study in order to fully elucidate MAPK pathways in regulating autophagy in early-stage OA. It was found that p-ERK1/2 was also increased in OA, while the ERK inhibitor, similar to JNK and p38 inhibitors, enhanced the autophagic process in OA.

To the best of our knowledge, the present study was the first to investigate the effect of MAPK signaling on autophagy in early-stage OA via intra-articular injection of specific MAPK inhibitors in rabbits. The results indicated that selective inhibition of the ERK, JNK, or p38 MAPK signaling pathways significantly increased the protein expression levels of autophagy biomarkers (Beclin1, ULK1 and LC3II/I) in OA model rabbits. Overall, these results suggested that the inhibition of MAPKs may restore impaired autophagy in OA. Therefore, MAPK inhibitors exerted a protective effect against OA by restoring impaired autophagy, suggesting a potential therapeutic strategy for $\mathrm{OA}$, as well as other degenerative disorders.

\section{Acknowledgements}

Not applicable.

\section{Funding}

No funding was received.

\section{Availability of data and materials}

The datasets generated and/or analyzed during the current study are available from the corresponding authors on reasonable request.

\section{Authors' contributions}

ZJY and CNL designed the study, CNL and JS conducted the experiments and JS and WJC analyzed the data. ZJY and WJC interpreted the results of the experiments. CNL generated the figures and drafted the manuscript and ZJY and JS edited and revised the manuscript. All authors reviewed and approved the final version of the manuscript. ZJY and JS confirm the authenticity of all the raw data.

\section{Ethics approval and consent to participate}

All animal experiments were approved by the Institutional Animal Care and Use Committee of The Second Xiangya Hospital of Central South University (Changsha, China; approval no. 2018005). All experimental procedures were performed in accordance with the Animal Research: Reporting of in vivo Experiments guidelines and the National Institutes of Health Guide for the Care and Use of Laboratory Animals.

\section{Patient consent for publication}

Not applicable.

\section{Competing interests}

The authors declare that they have no competing interests.

\section{References}

1. Mathiessen A and Conaghan PG: Synovitis in osteoarthritis: Current understanding with therapeutic implications. Arthritis Res Ther 19: 18, 2017.

2. Vila S: Inflammation in osteoarthritis. P R Health Sci J 36: 123-129, 2017.

3. Glyn-Jones S, Palmer AJ, Agricola R, Price AJ, Vincent TL, Weinans H and Carr AJ: Osteoarthritis. Lancet 386: 376-387, 2015.

4. Vina ER and Kwoh CK: Epidemiology of osteoarthritis: Literature update. Curr Opin Rheumatol 30: 160-167, 2018.

5. Saberi Hosnijeh F, Bierma-Zeinstra SM and Bay-Jensen AC: Osteoarthritis year in review 2018: Biomarkers (biochemical markers). Osteoarthritis Cartilage 27: 412-423, 2019.

6. Hunt MA, Charlton JM and Esculier JF: Osteoarthritis year in review 2019: Mechanics. Osteoarthritis Cartilage 28: 267-274, 2020.

7. Kanwar JR, Samarasinghe RM, Kumar K, Arya R, Sharma S, Zhou SF, Sasidharan S and Kanwar RK: Cissus quadrangularis inhibits IL-1beta induced inflammatory responses on chondrocytes and alleviates bone deterioration in osteotomized rats via p38 MAPK signaling. Drug Des Devel Ther 9: 2927-2940, 2015.

8. Bay-Jensen AC, Hoegh-Madsen S, Dam E, Henriksen K, Sondergaard BC, Pastoureau P, Qvist P and Karsdal MA: Which elements are involved in reversible and irreversible cartilage degradation in osteoarthritis? Rheumatol Int 30: 435-442, 2010.

9. Saha S, Panigrahi DP, Patil S and Bhutia SK: Autophagy in health and disease: A comprehensive review. Biomed Pharmacother 104: 485-495, 2018.

10. Lapaquette P, Guzzo J, Bretillon L and Bringer MA: Cellular and molecular connections between autophagy and inflammation. Mediators Inflamm 2015: 398483, 2015.

11. Deretic V, Saitoh T and Akira S: Autophagy in infection, inflammation and immunity. Nat Rev Immunol 13: 722-737, 2013.

12. Mizushima $\mathrm{N}$ and Komatsu M: Autophagy: Renovation of cells and tissues. Cell 147: 728-741, 2011.

13. Levine B and Kroemer G: Biological functions of autophagy genes: A disease perspective. Cell 176: 11-42, 2019.

14. Jeon H and Im GI: Autophagy in osteoarthritis. Connect Tissue Res 58: 497-508, 2017.

15. Luo P, Gao F, Niu D, Sun X, Song Q, Guo C, Liang Y and Sun W: The role of autophagy in chondrocyte metabolism and osteoarthritis: A comprehensive research review. Biomed Res Int 2019: 5171602, 2019.

16. Carames B, Taniguchi N, Otsuki S, Blanco FJ and Lotz M: Autophagy is a protective mechanism in normal cartilage, and its aging-related loss is linked with cell death and osteoarthritis. Arthritis Rheum 62: 791-801, 2010.

17. Filfan M, Sandu RE, Zavaleanu AD, GresiTa A, Glavan DG, Olaru DG and Popa-Wagner A: Autophagy in aging and disease. Rom J Morphol Embryol 58: 27-31, 2017.

18. Shi J, Zhang C, Yi Z and Lan C: Explore the variation of MMP3, JNK, p38 MAPKs, and autophagy at the early stage of osteoarthritis. IUBMB Life 68: 293-302, 2016.

19. Guo YJ, Pan WW, Liu SB, Shen ZF, Xu Y and Hu LL: ERK/ MAPK signalling pathway and tumorigenesis. Exp Ther Med 19: 1997-2007, 2020.

20. Plotnikov A, Zehorai E, Procaccia S and Seger R: The MAPK cascades: Signaling components, nuclear roles and mechanisms of nuclear translocation. Biochim Biophys Acta 1813: 1619-1633, 2011.

21. Xu K, Ma C, Xu L, Ran J, Jiang L, He Y, Adel Abdo Moqbel S, Wang $\mathrm{Z}$ and $\mathrm{Wu}$ L: Polygalacic acid inhibits MMPs expression and osteoarthritis via Wnt/beta-catenin and MAPK signal pathways suppression. Int Immunopharmacol 63: 246-252, 2018.

22. Kim EK and Choi EJ: Compromised MAPK signaling in human diseases: An update. Arch Toxicol 89: 867-882, 2015.

23. Mei S, Gu H, Ward A, Yang X, Guo H, He K, Liu Z and Cao W: p38 mitogen-activated protein kinase (MAPK) promotes cholesterol ester accumulation in macrophages through inhibition of macroautophagy. J Biol Chem 287: 11761-11768, 2012. 
24. Shen T, Miao Y, Ding C, Fan W, Liu S, Lv Y, Gao X, De Boevre M, Yan L, Okoth S, et al: Activation of the p38/MAPK pathway regulates autophagy in response to the CYPOR-dependent oxidative stress induced by zearalenone in porcine intestinal epithelial cells. Food Chem Toxicol 131: 110527, 2019.

25. He Y, She H, Zhang T, Xu H, Cheng L, Yepes M, Zhao Y and Mao Z: p38 MAPK inhibits autophagy and promotes microglial inflammatory responses by phosphorylating ULK1. J Cell Biol 217: 315-328, 2018.

26. Luo T, Zhang H, Yu Q, Liu G, Long M, Zhang K, Liu W, Song R, Bian J, Gu J, et al: ERK1/2 MAPK promotes autophagy to suppress ER stress-mediated apoptosis induced by cadmium in rat proximal tubular cells. Toxicol In Vitro 52: 60-69, 2018.

27. Barutcu SA, Girnius N, Vernia S and Davis RJ: Role of the MAPK/cJun NH2-terminal kinase signaling pathway in starvation-induced autophagy. Autophagy 14: 1586-1595, 2018.

28. Yan H, Gao Y and Zhang Y: Inhibition of JNK suppresses autophagy and attenuates insulin resistance in a rat model of nonalcoholic fatty liver disease. Mol Med Rep 15: 180-186, 2017.

29. He Q, Mei D, Sha S, Fan S, Wang L and Dong M: ERK-dependent mTOR pathway is involved in berberine-induced autophagy in hepatic steatosis. J Mol Endocrinol 59: X1, 2017. Erratum for: J Mol Endocrinol 57: 251-260, 2016.

30. Sridharan S, Jain K and Basu A: Regulation of autophagy by kinases. Cancers (Basel) 3: 2630-2654, 2011.

31. Webber JL and Tooze SA: Coordinated regulation of autophagy by p38alpha MAPK through mAtg9 and p38IP. EMBO J 29: 27-40, 2010.

32. Matsuzawa T, Fujiwara E and Washi Y: Autophagy activation by interferon- $\gamma$ via the p38 mitogen-activated protein kinase signalling pathway is involved in macrophage bactericidal activity. Immunology 141: 61-69, 2014.

33. Liu Y, Wang N, Zhang S and Liang Q: Autophagy protects bone marrow mesenchymal stem cells from palmitate-induced apoptosis through the ROS-JNK/p38 MAPK signaling pathways. Mol Med Rep 18: 1485-1494, 2018.

34. Zhu J, Yu W, Liu B, Wang Y, Shao J, Wang J, Xia K, Liang C, Fang W, Zhou C and Tao H: Escin induces Caspase-dependent apoptosis and autophagy through the ROS/p38 MAPK signalling pathway in human osteosarcoma cells in vitro and in vivo. Cell Death Dis 8: e3113, 2017.
35. NC3Rs Reporting Guidelines Working Group: Animal research: Reporting in vivo experiments: The ARRIVE guidelines. J Physiol 588: 2519-2521, 2010.

36. National Research Council (US) Committee for the Update of the Guide for the Care and Use of Laboratory Animals: Guide for the Care and Use of Laboratory Animals, 8th edition. National Academies Press (US), Washington, DC, 2011.

37. Sharma L: Osteoarthritis of the Knee. N Engl J Med 384: 51-59, 2021.

38. Hu XB, Kang RR, Tang TT, Li YJ, Wu JY, Wang JM, Liu XY and Xiang DX: Topical delivery of 3,5,4'-trimethoxy-trans-stilbene-loaded Microemulsion-based hydrogel for the treatment of osteoarthritis in a rabbit model. Drug Deliv Transl Res 9: 357-365, 2019.

39. Higashiguchi $\mathrm{T}$ and Go K: Effect of neurotropin on experimental osteoarthritis. Nihon Yakurigaku Zasshi 96: 153-1561, 1990 (In Japanese).

40. Pritzker KP, Gay S, Jimenez SA, Ostergaard K, Pelletier JP, Revell PA, Salter D and van den Berg WB: Osteoarthritis cartilage histopathology: Grading and staging. Osteoarthritis Cartilage 14: 13-29, 2006

41. van der Sluijs JA, Geesink RG, van der Linden AJ, Bulstra SK, Kuyer R and Drukker J: The reliability of the Mankin score for osteoarthritis. J Orthop Res 10: 58-61, 1992.

42. Waldstein W, Perino G, Gilbert SL, Maher SA, Windhager R and Boettner F: OARSI osteoarthritis cartilage histopathology assessment system: A biomechanical evaluation in the human knee. J Orthop Res 34: 135-140, 2016.

43. Dash S, Aydin Y and Moroz K: Chaperone-Mediated autophagy in the liver: Good or Bad? Cells 8: 1308, 2019.

This work is licensed under a Creative Commons Attribution-NonCommercial-NoDerivatives 4.0 International (CC BY-NC-ND 4.0) License. 